Rev. Elev. Méd, vét. Pays trop., 1978, 31 (4) : 437-442.

\title{
Contribution à l'étude de la fasciolose au Niger
}

\author{
par Pierre TAGER-KAGAN (*) \\ (avec la collaboration technique de Djibo GARBA (*), Labo RAHIOU (*), Nainou GUERO (*)
}

\begin{abstract}
RÉSUMÉ
Les zones à fasciolose se situent essentiellement dans les départements de Niamey (région Nord et moyenne du fleuve), de Dosso (régions des dallols) et le département de Diffa (région du lac Tchad).

La connaissance du cycle expérimental de $F$. gigantica et celle de la biologie de l'hôte intermédiaire Limnaea natalensis nous ont permis de cerner l'épidémiologie de la fasciolose, compte tenu des mouvements de troupeaux durant l'année autour des zones fasciologènes.

L'incidence de cette parasitose sur le développement de l'élevage Nigérien a été évaluée ; cette évaluation permettant d'envisager une prophylaxie médicale sous forme de campagnes de déparasitage régionales deux fois par an.
\end{abstract}

\section{INTRODUCTION}

Différentes enquêtes (Sondages coproscopiques, autopsies) $(8,9,10)$ ont montré sur une partie du cheptel Nigérien l'existence de la fasciolose à Fasciola gigantica; afin d'évaluer l'importance de cette trématodose dans la pathologie parasitaire $\mathrm{du}$ bétail, des études sur son épidémiologie ont été entreprises: elles devaient permettre de circonscrire les zones à distomatose, de préciser les modalités d'infestation du bétail, compte tenu des variations de population de l'hôte intermédiaire au cours de l'année: le bilan général de ces recherches aboutissant à l'établissement d'un plan de lutte contre cette helminthiase.

\section{IMPORTANCE ÉCONOMIQUE}

\section{Répartition géographique}

Les statistiques des pourcentages de foies

(*) Laboratoire de l'Elevage (I. N. R. A. N.) B. P. 485, Niamey (République du Niger). saisis pour distomatose par rapport au nombre d'animaux abattus (tabl. 1) montrent que c'est dans le département de Niamey que la fasciolose est la plus fréquente ; c'est surtout dans les régions Nord et moyenne du fleuve Niger que les saisies de foies sont les plus nombreuses (Tillabéry 57 p. 100 des foies de bovins saisis en 1975,56 p. 100 à Ayorou la même année).

Dans les départements de Dosso et Zinder, les saisies sont moins fréquentes; dans le département de Diffa, ce sont surtout les animaux abattus dans la région de N'Guigmi qui sont porteurs de lésions de distomatose, cela est en rapport avec la présence du Lac Tchad dans les eaux duquel l'hôte intermédiaire de $F$. gigantica se trouve en abondance.

Dans les départements de Tahoua, Maradi, Agades, la fasciolose n'a pas été signalée.

Les bovins, si l'on s'en réfère aux résultats de l'inspection sanitaire dans les abattoirs, sont proportionnellement plus touchés par la fasciolose que les petits ruminants; il y a, semble-t-il, deux raisons à cela : 
TABL. $\mathbb{N}^{\circ}$ I-Statiotiques des pourcentages de foies saisis pour distomatose par rapport au nombre d'animaux abattus.

\begin{tabular}{|c|c|c|c|c|c|c|c|c|c|}
\hline & \multicolumn{3}{|c|}{1973} & \multicolumn{3}{|c|}{1974} & \multicolumn{3}{|c|}{1975} \\
\hline & $\begin{array}{l}\text { Nombre } \\
\text { animaux } \\
\text { abattus }\end{array}$ & $\begin{array}{r}\text { Nombre } \\
\text { foies } \\
\text { saisis }\end{array}$ & Pourcentage & $\begin{array}{l}\text { Nombre } \\
\text { animaux } \\
\text { abattus }\end{array}$ & $\begin{array}{r}\text { Nombre } \\
\text { foies } \\
\text { saisis }\end{array}$ & Pourcentage & $\begin{array}{l}\text { Nombre } \\
\text { animaux } \\
\text { abattus }\end{array}$ & $\begin{array}{r}\text { Nombre } \\
\text { foies } \\
\text { saisis }\end{array}$ & Pourcentage \\
\hline $\begin{array}{l}\text { Départen } \\
\text { Niamey }\end{array}$ & & & & & & & & & \\
\hline Bovins & 5031 & 1086 & 21,5 & 3324 & 640 & 19,2 & 2324 & 596 & 25,6 \\
\hline Ovins & 11992 & 958 & 7,9 & 7163 & 554 & 7,7 & 6518 & 500 & 7,6 \\
\hline Caprins & 18473 & 710 & 3,8 & 17488 & 422 & 2,4 & 22357 & 599 & 2,6 \\
\hline $\begin{array}{l}\text { Départen } \\
\text { Zinder }\end{array}$ & & & & & & & & & \\
\hline Bovins & 8913 & 564 & 6,3 & 7552 & 274 & 3,6 & 3664 & 129 & 3,5 \\
\hline Ovins & 10734 & 278 & 2,5 & 9184 & 38 & 0,4 & 5838 & 7 & 0,1 \\
\hline Caprins & 23418 & 46 & 0,1 & 32139 & 22 & - & 28637 & 10 & - \\
\hline $\begin{array}{l}\text { Départem } \\
\text { Diffa }\end{array}$ & & & & & & & & & \\
\hline Bovins & 5216 & 527 & 10,1 & 3542 & 408 & 11,1 & 1675 & 270 & 16,1 \\
\hline Ovins & 1007 & 9 & 0,9 & 1084 & 34 & 3,1 & 1025 & 30 & 2,9 \\
\hline Caprins & 11751 & 79 & 0,6 & 10617 & 105 & 0,9 & 12236 & 89 & 0,7 \\
\hline $\begin{array}{l}\text { Départem } \\
\text { Dosso }\end{array}$ & & & & & & & & & \\
\hline Bovins & 2346 & 103 & 4,4 & 2180 & 259 & 11,8 & 878 & 126 & 14,3 \\
\hline Ovins & 2358 & 41 & 1,7 & 2712 & 60 & 2,2 & 1578 & 43 & 2,7 \\
\hline
\end{tabular}

Abattoir de Niamey non compris.

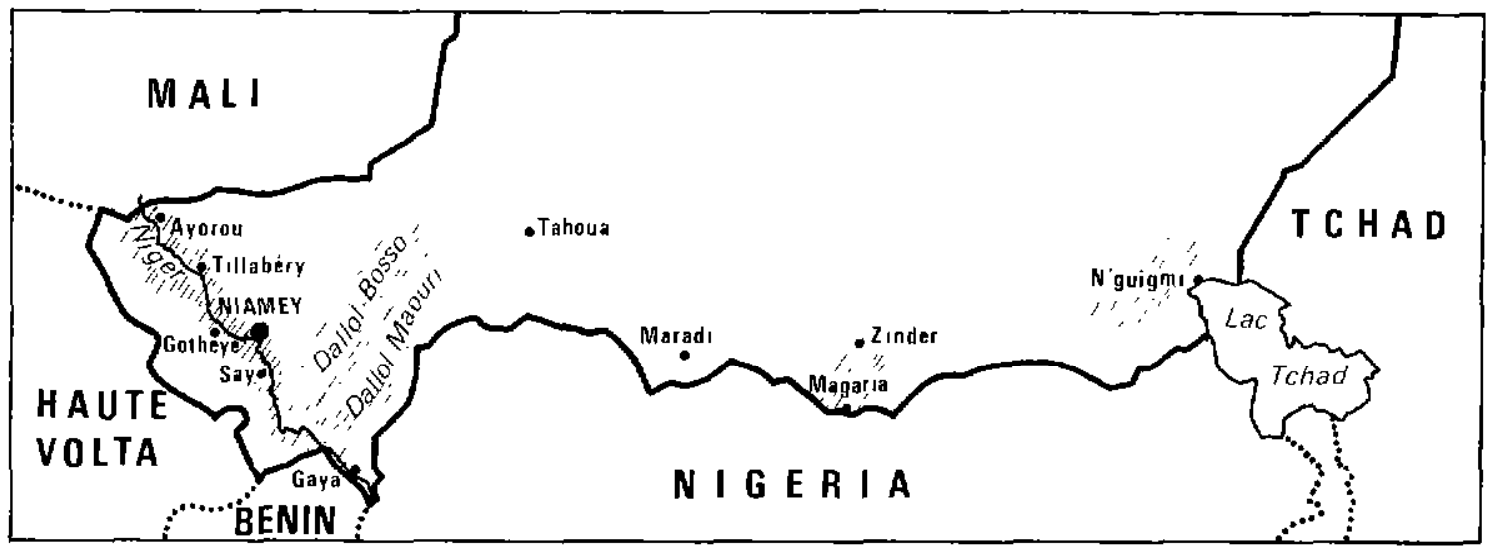

zone à distomatose $" \not /, " /, "$,

- premièrement les ovins développent plus souvent une fasciolose aiguë avec hémorragie hépatique mortelle échappant au recensement qui ne porte que sur les cas chroniques,

- deuxièmement il semblerait que les éle- veurs lâchent leurs troupeaux de zébus sur les zones fasciologènes avant les troupeaux de moutons ; les bovins plus résistants ingéreraient un maximum de métacercaires faisant ainsi diminuer le taux de contamination des moutons qui les suivent. 
En règle générale, l'infestation des bovins n'est jamais très importante ; sur les foies examinés chaque semaine à l'abattoir de Niamey, on trouve en moyenne une trentaine de fascioles par foie saisi ; lors de différentes expérimentations de médicaments, les autopsies pratiquées sur les animaux témoins ont également révélé des infestations pauci-parasitaires ( 22 fascioles/ foie lors d'une première expérience avec un maximum de $39 ; 51$ fascioles/foie lors d'un second essai avec un maximum de 124).

Cette pauci-infestation des bovins est liée à des phénomènes de résistance acquise ; cependant cette résistance n'est pas définitive, elle doit être entretenue par des réinfestations fréquentes (ce qui est habituellement le cas).

\section{Incidence de la faciolose sur l'élevage Nigé-} rien

Les estimations du cheptel nigérien en 1975 sont (10) :

$$
\begin{array}{ll}
\text { Bovins...... } & 2508000 \\
\text { Ovins ...... } & 2159000 \\
\text { Caprins .... } & 5395000
\end{array}
$$

Nos différentes enquêtes épidémiologiques nous ont montré l'existence de la fasciolose surtout dans le département de Niamey (arrondissement de Tillabery, Say, Niamey) de Dosso (arrondissement de Dosso, Birni N'Gaoure, Gaya) de Diffa (arrondissement de N'Guigm1).

\begin{tabular}{|c|c|c|c|}
\hline & Bovins & Ovins & Caprins \\
\hline Tillabery & 90000 & 130000 & 150000 \\
\hline & 83000 & 36000 & 33000 \\
\hline Niamey & 60000 & 20000 & 30000 \\
\hline Dosso. & 50000 & 20000 & 90000 \\
\hline Gaya ... & 35000 & 41000 & 83000 \\
\hline Birni. & 98000 & 28000 & 52000 \\
\hline \multirow[t]{2}{*}{ N'Guigmi ..... } & 70000 & 30000 & 120000 \\
\hline & 486000 & 305000 & 558000 \\
\hline
\end{tabular}

L'estimation du cheptel dans ces arrondissements est (10) :

Sur l'ensemble du cheptel nigérien c'est donc 19 p. 100 des bovins, 14 p. 100 des ovins et 10 p. 100 des caprins qui sont susceptibles d'être atteints de fasciolose.

Dans les départements à distomatose, nous avons recueilli les statistiques suivantes (pourcentage de foies saisis pour fasciolose par rapport au nombre d'animaux abattus).

$\begin{array}{ccc}\text { Bovins } & \text { Ovins } & \text { Caprins } \\ \text { (p. 100) } & (\text { p. 100) } & \text { (p. 100) }\end{array}$

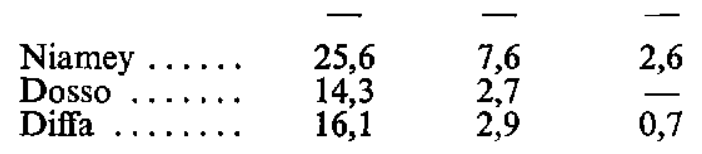

A la lecture de ces chiffres, il est certain que la fasciolose sur le plan national ne représente pas un problème pour le bon développement de l'élevage ; pour les bovins 19 p. 100 du cheptel nigérien parmi lesquels 18 p. 100 seulement ont présenté en 1975 des lésions de distomatose.

Mais, si on se place à l'échelle régionale, la fasciolose au niveau de certains arrondissements apparaît comme une parasitose majeure. (Tillabery 57 p. 100 des foies saisis, Ayorou 56 p. 100 , Kollo 34 p. 100, Birni N'Gaoure 28 p. 100 , N'Guigmi 17,5 p. 100 ).

La plupart du temps la fasciolose existe à l'état sub-clinique mais il n'en demeure pas moins que le pronostic économique peut être préoccupant :

- amaigrissement, croissance ralentie,

- diminution de la sécrétion lactée,

— saisies à l'abattage.

A Ayorou, on a effectué pendant plusieurs mois des examens coprologiques sur les veaux d'un même troupeau : en mai 14 p. 100 étaient porteurs de Fasciola, en août c'est 46 p. 100 des veaux qui étaient atteints; de même, après déparasitage des vaches du troupeau laitier de Say, on a trouvé six mois plus tard à l'examen coprologique des œufs de Fasciola sur 21 p. 100 de ces animaux.

Aussi, la discrétion de la symptomatologie de la fasciolose chez les bovins ne doit pas laisser croire à la bénignité économique de la maladie ; on peut même dire que cette discrétion clinique est un facteur aggravant car elle ne pousse pas les éleveurs à se préoccuper de cette helminthiase.

\section{II. ÉPIDÉMIOLOGIE}

\section{L'hôte intermédiaire $(3,6,7)$}

Des recherches faites sur le fleuve Niger de Ayorou jusqu'à Gaya nous ont permis de mettre en évidence Limnaea natalensis surtout dans la zone Nord et moyenne (Ayorou-Say); dans les 
criques où existe une végétation de nymphea, les limnées apparaissent en janvier, les populations restent peu importantes, elles atteignent leur maximum en mars-avril, puis elles décroissent selon le moment où le retrait des eaux intervient sur la végétation.

Dans les départements de Niamey et Dosso, soixante-seize mares ont été visitées; Limnaea natalensis n'a été trouvée que dans les Dallols Boboye et Maouri ; ne coulant qu'en saison des pluies, les dallols sont réduits en saison sèche à une série de mares occupant les bas-fonds plus ou moins imperméables; les populations de limnées y commencent à réapparaître en octobre ; leur nombre est important de janvier à avril avec un maximum vers février-mars; au fur et à mesure de la saison sèche, l'évaporation augmente la concentration en sels dissous du milieu et les conditions de multiplication et de survie s'amenuisent pour une faune malacologique dulçaquicole (4).

\section{Epidémiologie de la fasciolose}

\section{a) Sur le fleuve}

Les limnées recommencent à se multiplier en janvier, à ce moment les animaux pâturant le long du fleuve et parasités par $F$. Gigantica évacuent des cufs dans leur fèces; ces œufs, après leur transformation en miracidium, vont poursuivre leur développement dans des limnées.

L'cuf de $F$. gigantica $(1,2,7)$ atteignant le stade métacercaire infestante au bout de 55 à 80 jours, on peut considérer que les bovins revenant pâturer en mars sur les bords du fleuve s'infestent ; cette infestation se poursuivra jusqu'en mai ; au-delà, la diminution saisonnière des populations de mollusques rend plus difficile la contamination du bétail.

Il faut environ 90 à 100 jours pour que la métacercaire absorbée par l'animal donne un distome adulte, c'est donc fin mai, début juin

Epidêmiolagie de la fasciolose sur le fleuve Niger.

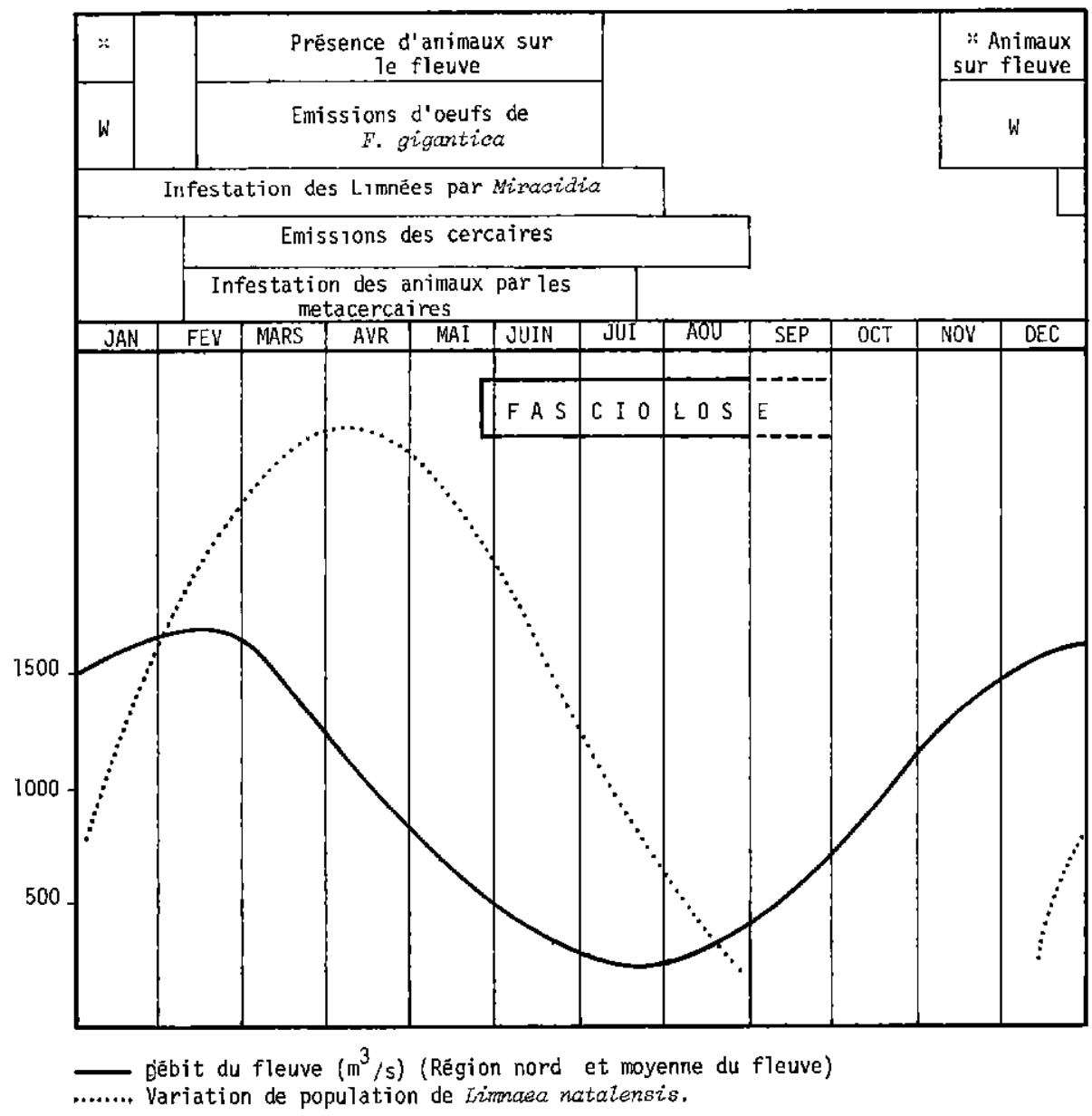


que les examens coproscopiques des bovins infestés début mars montreront des oufs de Fasciola gigantica.

Pour les animaux transhumant le long du fleuve, la distomatose est donc une maladie de fin de saison sèche, début de saison des pluies ; selon la date d'arrivée au bord du fleuve, la maladie apparaîtra fin mai, début juin jusqu'en août.

Pour les animaux sédentaires (troupeaux laitiers des différents villages le long du fleuve), la distomatose apparaîtra plus tôt (fin avrildébut mai).

\section{b) Sur les mares permanentes}

Durant la saison des pluies, les troupeaux transhument sur des pâturages inondés dans le Nord, ils commencent à gagner les pâturages des Dallols à partir de la mi-octobre ; l'infestation par les miracidia des limnées alors en voie de multiplication commence à cette époque, les miracidia provenant des œufs émis par les animaux porteurs de Fasciola pâturant autour des mares.

Dès la fin décembre, début janvier, les premières métacercaires sont disponibles et le bétail se trouvant autour des points d'eau se contaminera de janvier à fin avril ; début mai, la baisse des eaux et l'augmentation de la température entraînent la dessiccation et la mort des métacercaires ; en même temps, les populations de limnées diminuent considérablement. La contamination $\mathrm{du}$ bétail stoppe lorsque les animaux s'éloignent des mares permanentes en début de saison des pluies.

Compte tenu de la durée du cycle, la fasciolose fera sentir ses effets de la mi-mai à fin août.

\section{c) Sur le Lac Tchad}

Divers travaux (1) menés au Tchad ont montré que, dans cette zone, la distomatose est une maladie de fin de saison sèche, début de saison des pluies (15 mai-15 août).

\section{PROGRAMME DE LUTTE}

La prophylaxie sanitaire est des plus difficiles à réaliser sinon impossible :

- Impossibilité d'empêcher les animaux de venir pâturer dans les bourgoutières (au plus peut-on, comme le font les éleveurs Peulhs, retarder la venue des animaux les plus réceptifs (moutons) afin que leur infestation en métacercaires soit la moins importante possible) ;

- Impossibilité de traiter les zones nord et moyenne du fleuve par des agents molluscicides ;

- Difficulté de traiter les mares permanentes car elles servent le plus souvent à l'alimentation humaine et sont souvent poissonneuses.

La prophylaxie médicale s'avérera la plus efficace, elle consistera à maintenir le taux d'infestation le plus bas possible par des traitements systématiques.

Les efforts des services vétérinaires devront porter sur les arrondissements de Gaya et Birni (Dallols), sur l'arrondissement de N'Guigmi (Lac Tchad), et sur ceux de Tillabery, Say et Niamey (Région du fleuve).

A l'aide de l'un des fasciolicides modernes proposés par différents laboratoires pharmaceutiques, des traitements systématiques des animaux seront effectués en mai-juin quand les douves presque mûres peuvent être facilement touchées et détruites par les anthelminthiques. En octobre-novembre, on procédera à un autre traitement de manière à éliminer les fascioles adultes qui auraient résisté au premier traitement ; les animaux ainsi "stérilisés " ne contamineront pas leur lieu d'abreuvement où existent des populations de limnées ; cette intervention ne prendra son caractère prophylactique que dans la mesure où elle est généralisée à tous les animaux de la région considérée.

Au niveau des arrondissements intéressés, le service de l'élevage devra donc organiser régulièrement deux fois par an des campagnes de traitements antidistomiens (mai-juin et octobrenovembre) en attendant que les éleveurs euxmêmes prennent conscience des avantages économiques procurés par l'administration d'anthelminthique et qu'ils se décident à acheter et à administrer eux-mêmes le médicament.

\section{CONCLUSION}

La distomatose à Fasciola gigantica n'a pas une incidence économique importante sur l'élevage nigérien ; cependant, dans certains arron- 
dissements (Zone Nord et moyenne du fleuve Niger, régions des Dallols, alentours du Lac Tchad), le taux d'infestation est suffisamment important pour que les services de l'élevage envisagent des campagnes régionales de déparasitage.

\section{SUMMARY}

\section{Contribution to the study of fascioliasis in Niger}

Fascioliasis areas are essentially in the department of Niamey (in the North and middle region of the Niger river) in the department of Dosso (Dallols areas) and in the department of Diffa (Lake Tchad).

The knowledge of experimental cycle of Fasciola gigantica and the understanding of the biology of the intermediate host Limnaea natalensis have atlowed us to understand the epidemiology of fascioliasis taking into consideration of the movements of droves during the year around the fascioliasis areas.

Importance of this parasitism on the development of the Nigerian animal husbandry has been assessed ; this assessment allowing to consider a medical prophylaxis in the form of local campaign of treatment twice a year.

\section{RESUMEN}

\section{Contribución del estudio de la distomatosis en Niger}

Las zonas dónde se encuentra la distomatosis estan esencialmente en los departamentos de Niamey (región norte y media del río), de Dosso (regiones de los « dallols ») y de Diffa (región del lago Chad).

El conocimiento del ciclo experimental de $F$. gigantica y el de la biología del huesped intermediario Limnaea natalensis permitieron delimitar la epidemiología de la distomatosis, teniendo en cuenta los traslados de los rebaños durante el año alrededor de las zonas dónde reina dicha enfermedad.

Se evaluó la incidencia de esta parasitosis sobre el desarrollo de la ganaderia nigeriana, esta evaluación permitiendo proyectar una profilaxia medical bajo forma de campañas regionales de lucha contra los helmíntos dos veces al año.

\section{BIBLIOGRAPHIE}

1. BIRGI (E.), GRABER (M.). Mollusques pulmonés d'eau douce basommatophores vecteurs au Tchad d'affections parasitaires du bétail, leur élevage au laboratoire. Rev. Elev. Méd. vét. Pays trop., 1969, 22 (3) : 393-408.

2. DAYNES (P.). La distomatose à Madagascar. Cycle de Fasciola gigantica. Rev. Elev. Med. vét. Pays trop., 1967, 20 (4) 557-562.

3. EUZEBY (J.). Les maladies vermineuses des animaux domestiques. Tome II. $2^{\mathrm{e}}$ fasc., Livre I. Paris, Vigot Frères.

4. GRETILLAT (S.), GASTON (G.). Sur quelques particularités écologiques de la faune malacologique vectrice de trématodes dans les dallols Nigériens. Annls. Parasit. hum. comp., 1975, 50 (5) : 595-601.

5. GRIGORYAN (C. A.). Influence of external environmental factors on the biology and resistance of miracidia and metacercaria of F. gigantica, Veterinariyn, Moscou, 1956, 1, 9 B.

6. MANDAHL-BARTH (G.). Key to identification of east and central african freshwater snails of medical and veterinary importance. Bull. Wld Hlth Org., 1926, 27 (1) : 135-150.

7. TAGER-KAGAN (P.). Contribution à l'étude de l'épidémiologie des principales trématodoses des animaux domestiques dans la région du fleuve Niger. Rev. Elev. Med. vét. Pays trop., 1977, 30 (1) : 11-18.

8. TAGER-KAGAN (P.). Helminthes et helminthiases des animaux domestiques au Niger. Rapport Niamey, Laboratoire de l'Elevage, 1974.

9. TIBAYRENC (R.). Enquête helminthologique sur le fleuve Niger. Rapport Niamey, Laboratoire de l'Elevage, 1971.

10. Rapport annuel du Service de l'Elevage 1973, 1974, 1975 (Ministère de l’Economie Rurale, Niamey). 